\title{
On the Holonomic Systems of Linear Differential Equations, II *
}

\author{
Masaki Kashiwara \\ Massachusetts Institute of Technology, Department of Mathematics, \\ Cambridge, Massachusetts 02139, USA
}

In this paper we shall study the restriction of holonomic systems of differential equations.

Let $X$ be a complex manifold and $Y$ a submanifold, and let $\mathcal{O}_{X}$ and $\mathscr{D}_{X}$ be the sheaf of the holomorphic functions and the sheaf of the differential operators of finite order, respectively. If a function $u$ on $X$ satisfies a system of differential equations, the restriction of $u$ onto $Y$ also satisfies the system of differential equations derived from the system on $X$. This leads to the following definition. Let $\mathscr{M}$ be a $\mathscr{D}_{X}$-Module. The restriction of $\mathscr{M}$ onto $Y$ is, by definition, $\mathcal{O}_{Y} \otimes \mathscr{M}$. In [4] it is proved that if $\mathscr{M}$ is a coherent $\mathscr{D}_{X}$-Module and if $Y$ is noncharacteristic to $\mathscr{M}$, then the restriction of $\mathscr{M}$ is also a coherent $\mathscr{D}_{Y}$-Module. However, if $Y$ is characteristic, the restriction is no longer coherent in general. For examples, if $X=\mathbb{C}^{n}$ and $Y=\left\{x=\left(x_{1}, \ldots, x_{n}\right) \in X ; x_{1}=0\right\}$ and $\mathscr{M}=\mathscr{D}_{X}$, the restriction $\mathscr{M} / x_{1} \mathscr{M}$ is a free $\mathscr{D}_{Y}$-Module generated by $D_{1}^{m}(m=0,1,2, \ldots)$ and is not coherent.

We shall prove the following theorems in this paper.

Theorem. Let $\mathscr{M}$ be a holonomic $\mathscr{D}_{X}$-Module on a complex manifold $X$ and $f$ a holomorphic map from $Y$ to $X$. Then $\mathcal{O}_{Y} \underset{f^{-1} \mathcal{O}_{X}}{\otimes} f^{-1} \mathscr{M}$ is a holonomic system on $Y$.

This theorem is proved by Bernstein [1] in the polynomial case.

At the same time, we shall prove

Theorem. If $\mathscr{M}$ is a holonomic $\mathscr{D}_{X}$-Module, and if $\mathscr{I}$ is a coherent Ideal of $\mathscr{O}_{X}$, then $\lim _{m} \mathscr{E} x t_{\mathscr{O}_{X}}^{k}\left(\mathcal{O}_{X} / \mathscr{I}^{m} ; \mathscr{M}\right)$ are also holonomic $\mathscr{D}_{X}$-Modules.

Theorem. If $\mathscr{M}$ is a holonomic $\mathscr{D}_{X}$-Module defined on $X$ and holonomic outside an analytic subset $Y$, then $\mathscr{M} / \mathscr{H}_{\mathrm{Y}}^{0}(\mathscr{M})$ is holonomic on $X$.

These theorems imply in particular the following: Let $\mathscr{F}$ be a coherent $\mathcal{O}_{X}$-Module and let $\nabla$ be a meromorphic integrable connection on $\mathscr{F}$ with a pole

$\star \quad$ This is the second of the series of papers which are concerned with holonomic systems. The paper [5] is the first of this series 
on a hypersurface $Y$. Then, $\mathscr{H}_{[X \mid Y]}^{0}(\mathscr{F})$ (i.e., the sheaf of the meromorphic sections of $\mathscr{F}$ with a pole on $Y$ ) is a holonomic $\mathscr{D}_{X}$-Module (in particular, coherent).

Also, we shall prove the following theorem.

Theorem. For two holonomic $\mathscr{D}_{X}$-Modules $\mathscr{M}$ and $\mathscr{N}, \mathscr{E} x t^{j}(\mathscr{M} ; \mathcal{N})$ are constructible (i.e., $\operatorname{dim}_{\mathbb{C}} \mathscr{E} x t^{j}(\mathscr{M} ; \mathscr{N})_{x}<\infty$ for any $x \in X$ and there is a stratification on $X$ on each of whose stratum $\mathscr{E} x t_{\mathscr{Q}}^{j}(\mathscr{M}, \mathscr{N})$ is locally constant).

However, the author does not know how to stratify $X$ so that $\mathscr{E} x t_{\mathscr{Q}}^{j}(\mathscr{M}, \mathscr{N})$ is constructible on the strata. This problem is tightly connected with the problem of determining the characteristic variety of $\mathcal{O}_{Y} \otimes \mathscr{M}$

I wish to thank J.E. Björk, J.-M. Kantor and B.Malgrange for their kind suggestions and friendly discussions about these subjects.

\section{§ 1. Algebraic Local Cohomologies}

1.1. In this paper we denote by $X$ a complex manifold, by $\mathscr{O}_{X}$ the sheaf of the holomorphic functions on $X$ and by $\mathscr{D}_{X}$ the sheaf of the linear differential operators of finite order.

1.2. Let $\mathscr{I}$ be a coherent $\mathcal{O}_{X}$-Ideal and $Y$ the support of $\mathcal{O}_{X} / \mathscr{I}$. For an $\mathcal{O}_{x}$-Module $\mathscr{F}$, we define with $[2,3]$

(1.2.1) $\quad \Gamma_{[X \mid Y]}(\mathscr{F})=\underset{m}{\lim } \mathscr{H}_{\operatorname{lom}_{\mathscr{O}_{X}}}\left(\mathscr{I}^{m} ; \mathscr{F}\right)$,

(1.2.2) $\quad \Gamma_{[Y]}(\mathscr{F})=\underset{\vec{m}}{\lim } \mathscr{H}_{\text {om }}{ }_{O_{X}}\left(\mathcal{O}_{X} / \mathscr{I}^{m} ; \mathscr{F}\right)$.

This definition depends only on $Y$ (not on the choice of $\mathscr{I}$ ). We have an exact sequence:

$$
0 \rightarrow \Gamma_{[Y]}(\mathscr{F}) \rightarrow \mathscr{F} \rightarrow \Gamma_{[X \mid Y]}(\mathscr{F})
$$

Lemma 1.1. If $\mathscr{F}$ is a $\mathscr{D}_{X}$-Module, $\Gamma_{[X \mid Y]}(\mathscr{F})$ and $\Gamma_{[Y]}(\mathscr{F})$ have a structure of $\mathscr{D}_{X}$-Modules so that (1.2.3) is $\mathscr{D}_{X}$-linear.

Proof. We have evidently

$$
\Gamma_{[X \mid Y]}(\mathscr{F})=\underset{m}{\lim } \mathscr{H}_{\operatorname{lom}_{\mathscr{D}_{X}}}\left(\mathscr{D}_{X} \mathscr{F}^{m} ; \mathscr{F}\right)
$$

and

$$
\Gamma_{[Y]}(\mathscr{F})=\lim _{m} \mathscr{H} \operatorname{osm}_{\mathscr{D}_{X}}\left(\mathscr{D}_{X} / \mathscr{D}_{X} \mathscr{I}^{m} ; \mathscr{F}\right)
$$

because $\mathscr{D}_{X}$ is faithfully flat over $\mathcal{O}_{X}$.

We shall define the multiplication of a differential operator $P$ with $\Gamma_{[X \mid Y]}(\mathscr{F})$. Suppose that $P$ is of order $\leqq l$. Then we have

$$
\mathscr{D}_{X} \mathscr{I}^{m} P \subset \mathscr{D}_{X} \mathscr{I}^{m-l} \quad \text { for } m \geqq l .
$$


This gives the $\mathscr{D}_{X}$-linear homomorphism

$$
\mathscr{D}_{X} \mathscr{I}^{m} \rightarrow \mathscr{D}_{X} \mathscr{I}^{m-1}
$$

by the multiplication of $P$. Hence, we get the homomorphism

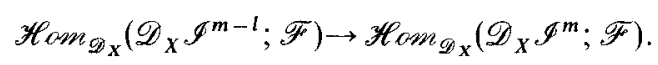

Taking the inductive limit on $m$, we have the homomorphism $\Gamma_{[X \mid Y]}(\mathscr{F}) \rightarrow \Gamma_{[X \mid Y]}(\mathscr{F})$, which will be the multiplication by $P$. It is easy to check that this gives a structure of $\mathscr{D}_{X}$-Module on $\Gamma_{[X \mid Y]}(\mathscr{F})$ and that $\mathscr{F} \rightarrow \Gamma_{[X \mid Y]}(\widetilde{\mathscr{F}})$ is $\mathscr{D}_{X}$-linear. Therefore, the kernel $\Gamma_{[Y]}(\mathscr{F})$ of this homomorphism has also a structure of $\mathscr{D}_{X}$-Module.

We shall denote by $\mathscr{H}_{[X \mid Y]}^{k}(\mathscr{F})$ (resp. $\mathscr{H}_{[Y]}^{k}(\mathscr{F})$ ) the $k$-th derived functor of $\Gamma_{[X \mid X]}(\mathscr{F})\left(\right.$ resp. $\left.\Gamma_{[Y]}(\mathscr{F})\right)$. have

Since a stalk of an injective $\mathscr{D}_{X}$-Module is injective over a stalk of $\mathcal{O}_{X}$, we

$$
\begin{aligned}
& \mathscr{H}_{[X \mid Y]}^{k}(\mathscr{\mathscr { F }})={\underset{m}{m}}_{\lim } \mathscr{E} x t_{\mathcal{O}_{X}}^{k}\left(\mathscr{I}^{m} ; \mathscr{F}\right) \\
& \mathscr{H}_{[Y]}^{k}(\mathscr{F})=\underset{\underset{m}{\lim } \mathscr{E}}{\mathscr{E}} x t_{\mathcal{O}_{X}}^{k}\left(\mathscr{O}_{X} / \mathscr{I}^{m} ; \mathscr{F}\right) .
\end{aligned}
$$

We denote by $\mathbb{R} \Gamma_{[Y]}, \mathbb{R} \Gamma_{[X \mid Y]}$ the right derived functor in the derived category. We have the following triangles:

$$
\begin{aligned}
& \mathbb{R} \Gamma_{[Y]} \mathscr{F} \\
& \overbrace{\mathscr{F}}^{\longrightarrow} \longrightarrow \mathbb{R} \Gamma_{[X \mid Y]}\left(\mathscr{F}^{*}\right), \\
& \mathbb{R} \Gamma_{\left[X \mid Y_{1} \cap Y_{2}\right]}(\mathscr{F}) \\
& \mathbb{R} \Gamma_{\left[X \mid Y_{1}\right]}\left(\mathscr{F}^{*}\right) \oplus \mathbb{R} \Gamma_{\left[X \mid Y_{2}\right]^{(}}\left(\mathscr{F}^{*}\right) \rightarrow \mathbb{R} \Gamma_{\left.\left[X \mid Y_{1} \cup Y_{2}\right]^{(\mathscr{F}}\right)}\left(\tilde{\mathscr{F}^{*}}\right)
\end{aligned}
$$

and we have also the relations

$$
\begin{aligned}
\mathbb{R} \Gamma_{\left[Y_{1} \cap Y_{2}\right]}(\mathscr{F}) & =\mathbb{R} \Gamma_{\left[Y_{1}\right]} \mathbb{R} \Gamma_{\left[Y_{2}\right]}(\mathscr{F}), \\
\mathbb{R} \Gamma_{\left[X \mid Y_{1}\right]} \mathbb{R} \Gamma_{\left[Y_{2}\right]}(\mathscr{F}) & =\mathbb{R} \Gamma_{\left[Y_{2}\right]} \mathbb{R} \Gamma_{\left[X \mid Y_{1}\right]}(\mathscr{F}), \\
\mathbb{R} \Gamma_{[X \mid Y]} \mathbb{R} \Gamma_{\left[Y Y^{*}\right.}(\mathscr{F}) & =\mathbb{R} \Gamma_{[Y]} \mathbb{R} \Gamma_{\left[X \mid Y_{1}\right]}\left(\mathscr{F}^{*}\right)=0 . \\
\mathbb{R} \Gamma_{\left[X \mid Y_{1}\right]} \mathbb{R} \Gamma_{\left[X \mid Y_{2}\right]}(\mathscr{F}) & =\mathbb{R} \Gamma_{\left[X \mid Y_{1} \cup Y_{2}\right]}(\mathscr{F}) .
\end{aligned}
$$

1.3. Suppose $Y$ is a hypersurface defined by $f=0$ with a holomorphic function $f$. For an $\mathscr{O}_{X}$-Module $\mathscr{F}$, we shall denote by $\mathscr{F}_{f}$ the $\mathscr{O}_{X}$-Module associated with the presheaf $U \mapsto \Gamma(U ; \mathscr{F})_{f}$; here $\Gamma(U ; \mathscr{F})_{f}$ is a localization by $f$. Then it is easy to see that

$$
\mathbb{R} \Gamma_{[X \mid Y]}(\mathscr{F})=\mathscr{F}_{f}=\mathcal{O}_{X_{f}} \otimes \mathscr{F}
$$


$\mathscr{D}_{X, j}$ is nothing but the Ring of differential operators with pole on $Y$. Although $\mathscr{D}_{X}$ has two structures of $\mathscr{O}_{X}$-Modules (by the left and the right multiplications), we obtain the same $\Gamma_{[X \mid Y]}\left(\mathscr{D}_{X}\right)$.

1.4. We shall investigate the meaning of $\Gamma_{[X \mid Y]}$ and $\Gamma_{[Y]}$ from the viewpoint of systems of differential equations.

Theorem 1.2. Let $\mathscr{F}^{\cdot}$ be a complex of right $\mathscr{D}_{X}$-Modules and $\mathscr{G}^{\circ}$ a complex of left $\mathscr{D}_{X}$-Modules. Then, for any analytic subset $Y$, we have

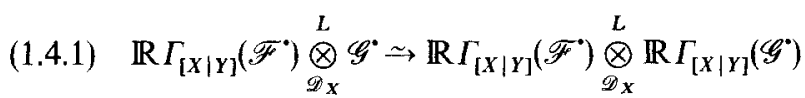

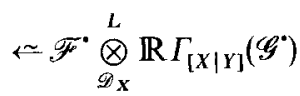

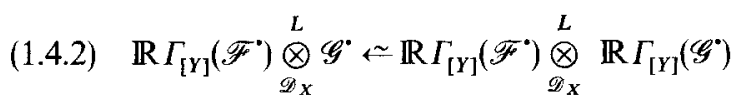

$$
\begin{aligned}
& \stackrel{\mathscr{P}}{\rightarrow} \mathscr{\mathscr { D }}_{\boldsymbol{X}}^{L} \mathbb{R} \Gamma_{[Y]}(\mathscr{G}) .
\end{aligned}
$$

Here $\stackrel{L}{\otimes}$ is the left derived functor of $\otimes$ in the derived category.

Proof. First we shall observe that (1.4.1) and (1.4.2) are equivalent. In fact, if (1.4.1) holds, then

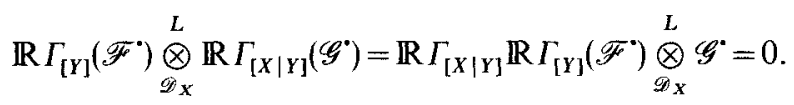

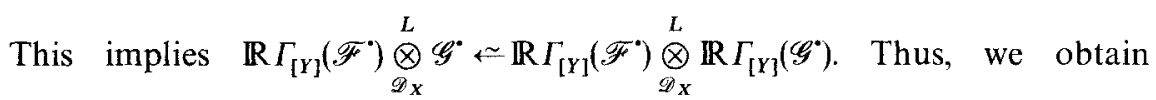
(1.4.2.). Conversely, if (1.4.2) holds, then

$$
\mathbb{R} \Gamma_{[X \mid Y]}\left(\mathscr{F}^{\cdot}\right) \underset{\mathscr{D}_{X}}{\stackrel{L}{\otimes}} \mathbb{R} \Gamma_{[Y]}(\mathscr{G})=\mathbb{R} \Gamma_{[Y]} \mathbb{R} \Gamma_{[X \mid Y]}(\mathscr{F} \cdot \underset{\mathscr{P}}{\stackrel{L}{\otimes}} \mathscr{G}=0,
$$

which implies (1.4.1).

Now, we shall prove this theorem. The question being local, we may assume that $Y$ is a finite intersection of hypersurfaces $Y_{1}, \ldots, Y_{l}$. We shall prove it by induction on $l$.

a) When $l=1$ (i.e., $Y$ is a hypersurface), suppose that $Y$ is defined by $f=0$. We may assume that any stalk $\mathscr{F}_{x}^{j}$ and $\mathscr{G}_{x}^{j}$ are free $\mathscr{D}_{X, x}$-modules. Thus, it is enough to show (1.4.1) when $\mathscr{F}=\mathscr{D}_{X}$ and $\mathscr{G}=\mathscr{D}_{X}$. Then we have $\mathbb{R} \Gamma_{[X \mid Y]}(\mathscr{F})$ $=\mathscr{D}_{X, f}$ and $\mathbb{R} \Gamma_{[X \mid Y]}(\mathscr{G})=\mathscr{D}_{X, f}$. We have also $\mathscr{D}_{X, f} \stackrel{\mathscr{D}}{\mathscr{D}}^{\stackrel{L}{\otimes} \mathscr{D}_{X, f}=\mathscr{D}_{X, f} .}$. This shows
(1.4.1).

b) When $l \geqq 2$. Set $Y^{\prime}=Y_{2} \cap \ldots \cap Y_{l}$. By the hypothesis of the induction, the theorem is true for $Y^{\prime}$. Therefore, we have 


$$
\begin{aligned}
& \mathbb{R} \Gamma_{[Y]}\left(\mathscr{F}^{*}\right) \underset{\mathscr{Z}_{X}}{\stackrel{L}{\otimes}} \mathscr{G}^{*} \\
& =\mathbb{R} \Gamma_{\left[Y_{1}\right]} \mathbb{R}_{\left[Y^{\prime}\right]}(\mathscr{F}) \underset{\mathscr{Q}_{X}}{\otimes} \mathscr{G}=\mathbb{R} \Gamma_{\left[Y^{\prime}\right]}(\mathscr{F}) \stackrel{L}{\otimes} \mathbb{R} \Gamma_{\left[Y_{X}\right]}\left(\mathscr{G}^{*}\right) \\
& =\mathscr{F}^{\circ} \stackrel{L}{\otimes} \mathbb{R} \Gamma_{\left[Y^{\prime}\right]} \mathbb{R} \Gamma_{\left[Y_{1}\right]}\left(\mathscr{G}^{*}\right)=\mathscr{\mathscr { F }} \cdot \stackrel{L}{\otimes} \mathbb{R} \Gamma_{\left[Y^{\prime}\right]}\left(\mathscr{G}^{*}\right) .
\end{aligned}
$$

This shows (1.4.2). Q.E.D.

We shall prove the following two theorems in this paper.

Theorem 1.3. Let $Y$ be an analytic subset of a complex manifold $X$, and $\mathscr{M}$ a coherent $\mathscr{D}_{X}$-Module which is holonomic on $X-Y$. Then $\mathscr{H}_{[X \mid Y]}^{k}(\mathscr{M})$ are holonomic $\mathscr{D}_{X}$-Modules.

Theorem 1.4. Under the same assumption as above, if $\mathscr{M}$ is holonomic on $X$, then $\mathscr{H}_{[Y]}^{\mathrm{k}}(\mathscr{M})$ are holonomic $\mathscr{D}_{X}$-Modules.

Together with Theorem 1.2, we have the following theorem.

Theorem 1.5. Let $Y$ be an analytic subset of a complex manifold $X, \mathscr{M}$ a coherent $\mathscr{D}_{X}$-Module and $\mathscr{N}$ a $\mathscr{D}_{X}$-Module.

a) If $\mathscr{M}$ is holonomic on $X-Y$, then

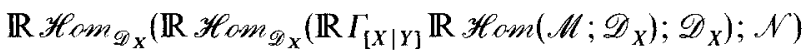

$$
\begin{aligned}
& =\mathbb{R} \mathscr{H}_{\text {om }} \mathscr{D}_{X}\left(\mathscr{M} ; \mathbb{R} \Gamma_{[X \mid Y]}(\mathcal{N})\right) .
\end{aligned}
$$

b) If $\mathscr{M}$ is holonomic on $X$, then

$$
\begin{aligned}
& \mathbb{R} \mathscr{H} O m_{\mathscr{D}_{X}}\left(\mathbb{R} \mathscr{H} \operatorname{mom}_{\mathscr{D}_{X}}\left(\mathbb{R} \Gamma_{[Y]} \mathbb{R} \mathscr{H} \operatorname{osm}\left(\mathscr{M} ; \mathscr{D}_{X}\right) ; \mathscr{D}_{X}\right) ; \mathscr{N}\right) \\
& =\mathbb{R} \mathscr{H} o m_{\mathscr{O} X}\left(\mathscr{M} ; \mathbb{R} \Gamma_{[Y]}(\mathscr{N})\right) \text {. }
\end{aligned}
$$

Proof. Let us prove a). We have

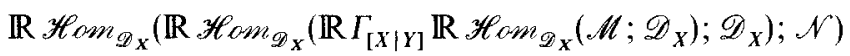

$$
\begin{aligned}
& =\mathbb{R} \Gamma_{[X \mid Y]} \mathbb{R} \mathscr{H}_{\operatorname{Oam}_{\mathscr{D}_{X}}}\left(\mathscr{M} ; \mathscr{D}_{X}\right) \stackrel{L}{\otimes} \mathscr{\mathscr { Z } X} \\
& =\mathbb{R} \mathscr{H} \operatorname{com}_{\mathscr{D}_{X}}\left(\mathscr{M} ; \mathscr{D}_{X}\right) \stackrel{L}{\stackrel{L}{\mathscr{D}}_{X}} \mathbb{R} \Gamma_{[X \mid Y]}(\mathscr{N}) \\
& =\mathbb{R} \mathscr{H}_{o m_{\mathscr{D}_{X}}}\left(\mathscr{M} ; \mathbb{R} \Gamma_{[X \mid Y]}(\mathscr{N})\right) .
\end{aligned}
$$

b) is obtained in the same way. Q.E.D.

Remark. In [7] we will see that if $\mathscr{M}$ has regular singularity, then $\mathscr{D}^{\infty} \otimes \mathbb{R} \Gamma_{[Y]}(\mathscr{M})=\mathbb{R} \Gamma_{Y}\left(\mathscr{D}^{\infty} \otimes \mathscr{M}\right)$, where $\mathscr{D}^{\infty}$ is the sheaf of the differential operators of infinite order. However, this relation does not hold when $\mathscr{M}$ has irregular singularity.

1.5. Let $\Theta$ be the sheaf of the vector fields. Then $\mathscr{D}_{X}$ is an $\mathcal{O}_{X}$-Algebra generated by $\Theta$. Therefore, it is easy to see the following lemma. 
Lemma 1.6. Let $\mathscr{F}$ be an $\mathcal{O}_{X}$-Module. Suppose that a sheaf homomorphism $\psi: \Theta \otimes \mathscr{F} \rightarrow \mathscr{F}$ satisfies the following conditions:

(i) $\psi(a v \otimes s)=a \psi(v \otimes s)$ (resp. $\psi(a v \otimes s)=\psi(v \otimes a s)$ for $a \in \mathcal{O}_{X}, \quad v \in \Theta_{X}$ and $s \in \mathscr{F}$.

(ii) $\psi(v \otimes a s)=a \psi(v \otimes a)+v(a) \psi(v \otimes s)(\operatorname{resp} . \psi(a v \otimes s)=a \psi(v \otimes s)$

$-v(a) \psi(v \otimes s))$ for $a \in \mathcal{O}_{X}, v \in \Theta_{X}$ and $s \in \mathscr{F}$.

(iii) $\psi\left(\left[v_{1} v_{2}\right] \otimes s\right)=\psi\left(v_{1} \otimes \psi\left(v_{2} \otimes s\right)\right)-\psi\left(v_{2} \otimes \psi\left(v_{1} \otimes s\right)\right)($ resp. $\psi\left(\left[v_{1}, v_{2}\right] \otimes s\right)=\psi\left(v_{2} \otimes \psi\left(v_{1} \otimes s\right)\right)-\psi\left(v_{1} \otimes \psi\left(v_{2} \otimes s\right)\right)$ for $v_{1}, v_{2} \in \Theta_{X}$ and $s \in \mathscr{F}$.

Then there is a unique structure of the left (resp. right) $\mathscr{D}_{X}$-Module on $\mathscr{F}$ such that $\psi(v \otimes s)=v s$ (resp. $\psi(v \otimes s)=s v)$ and that the induced structure of the $\mathscr{O}_{X^{-}}$ Module coincides with the original one of $\mathscr{F}$.

1.6. Let $\mathscr{M}$ and $\mathscr{N}$ be two left $\mathscr{D} x$-Modules. Then $\mathscr{M} \otimes \mathscr{N}$ has the structure of a left $\mathscr{D}_{X}$-Module by $v(s \otimes t)=v s \otimes t+s \otimes v t$ for $v \in \Theta_{X}^{\mathscr{G}_{X}}, s \in \mathscr{M}, t \in \mathscr{N}$. If $\mathscr{M}$ is a right $\mathscr{D}_{X}$-Module and $\mathscr{N}$ is a left $\mathscr{D}_{X}$-Module, $\mathscr{M} \otimes \mathscr{N}$ has the structure of a right $\mathscr{D}_{X}$-Module by $(s \otimes t) v=s v \otimes t-s \otimes v t$. If $\mathscr{M}$ and $\mathscr{N}$ are right $\mathscr{D}_{X^{-}}$ Modules, then $\mathscr{H}_{0 m_{\mathcal{O}_{X}}}(\mathscr{M} ; \mathscr{N})$ has the structure of a left $\mathscr{D}_{X}$-Module by $(v f)(s)$ $=f(s v)-f(s) v$ for $f \in \mathscr{H}_{0_{2} \mathscr{M}_{X}}(\mathscr{M} ; \mathscr{N}), v \in \Theta_{X}$ and $s \in \mathscr{M}$. If $\mathscr{M}$ is a left $\mathscr{D}_{X}$-Module and $\mathscr{N}$ is a right $\mathscr{D}_{X}$-Module, then $\mathscr{H}^{\circ} \mathfrak{o n}_{\mathscr{O}_{X}}(\mathscr{M} ; \mathscr{N})$ has the structure of a right $\mathscr{D}_{X}$-Module by $(f v)(s)=f(v s)+f(s) v$ for $f \in \mathscr{H} \operatorname{mom}_{\mathscr{O}_{X}}(\mathscr{M} ; \mathscr{N}) . v \in \Theta_{X}$ and $s \in \mathscr{M}$.

These facts are easily checked by using Lemma 1.6. Since the sheaf $\Omega_{X}^{n}$ of the $n$-forms $(n=\operatorname{dim} X)$ is a right $\mathscr{D}_{X}$-Module, $\mathscr{M} \mapsto \Omega_{X}^{n} \otimes \mathscr{M}$ and $\mathscr{N} \mapsto \mathscr{H}^{\prime} m_{C_{X}}$ $\left(\Omega_{X}^{n} ; \mathcal{N}\right)$ give the equivalence of the category of left $\mathscr{D}_{X}$-Modules and the category of right $\mathscr{D}_{X}$-Modules.

The following lemma being easily checked, we leave the proof to the reader.

Lemma 1.7. (i) Let $\mathscr{M}$ be a right (resp. left) $\mathscr{D}_{X}$-Module, $\mathscr{N}$ a left (resp. right) $\mathscr{D}_{X}$-Module and $\mathscr{L}$ a right $\mathscr{D}_{X}$-Module. Then

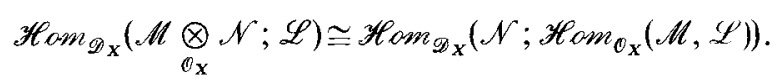

(ii) If $\mathscr{M}$ is a right $\mathscr{D}_{X}-$ Module and if $\mathscr{N}$ and $\mathscr{L}$ are left $\mathscr{D}_{X}$-Modules, then

$$
(\mathscr{M} \otimes \mathscr{O}) \underset{\mathscr{O}_{X}}{\otimes} \mathscr{L} \cong \mathscr{M} \underset{\mathscr{D}_{X}}{\otimes}\left(\mathcal{N} \otimes \underset{\mathcal{O}_{x}}{\otimes} \mathscr{L}\right)
$$

Lemma 1.8. Let $\mathscr{M}$ (resp. $\mathscr{N}^{\bullet}$ ) be a complex of right (resp. left) $\mathscr{D}_{X}$-Modules. Then

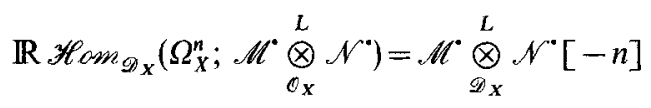

where $n=\operatorname{dim} X$.

Proof. We have 


$$
\begin{aligned}
& \mathbb{R} \mathscr{H}_{0 m_{\mathscr{D}}}\left(\Omega_{X}^{n} ; \mathscr{M}^{\cdot} \underset{\mathscr{O}_{X}}{\otimes} \mathscr{N}^{\cdot}\right) \\
& =\left(\mathscr{M}^{\cdot} \underset{\mathscr{U}_{X}}{\otimes} \mathscr{N}^{\cdot}\right)_{\mathscr{D}_{X}}^{\otimes_{L}^{L}} \mathbb{R} \mathscr{H} \operatorname{am}\left(\Omega_{X}^{n} ; \mathscr{D}_{X}\right) \\
& =\left(\mathscr{M}^{\bullet} \otimes_{\mathscr{O}_{X}}^{L} \mathscr{N}\right) \underset{\mathscr{D}_{X}}{\otimes} \mathcal{O}_{X}[-n] \\
& =\mathscr{M}^{\cdot}{\underset{\mathscr{D}}{\mathrm{X}}}^{\mathrm{L}}\left(\mathcal{N}^{*} \otimes_{\mathscr{O}_{\mathrm{X}}}^{\mathcal{O}_{X}}\right)[-n] \\
& =\mathscr{M}^{\stackrel{L}{\otimes} \mathscr{\mathscr { A }}_{X}} \mathscr{N}^{\cdot}[-n] . \quad \text { Q.E.D. }
\end{aligned}
$$

Lemma 1.9. For a coherent left $\mathscr{D}_{X}$-Module $\mathscr{M}$ and a $\mathscr{D}_{X}$-Module $\mathscr{N}$,

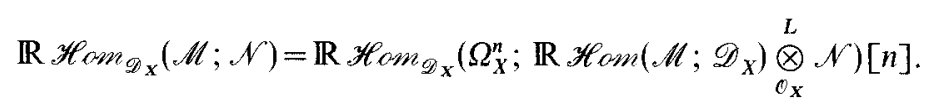

where $n=\operatorname{dim} X$, and $\Omega_{X}^{n}$ is the sheaf of $n$-forms on $X$.

In fact, we have

$\mathbb{R} \mathscr{H}_{\operatorname{osm}_{\mathscr{D}}}(\mathscr{M} ; \mathscr{N})=\mathbb{R} \mathscr{H} \operatorname{am}\left(\mathscr{H} ; \mathscr{D}_{X}\right) \stackrel{L}{\otimes} \mathscr{N}$

\section{§ 2. $b$-Functions}

2.1. Let $f$ be a holomorphic function on $X$ and $Y$ the zeros of $f$. As we mentioned, $\mathscr{M}_{f}$ is not necessarily a coherent $\mathscr{D}_{X}$-Module even if $\mathscr{M}$ is a coherent $\mathscr{D}_{X}$-Module. We shall show that $\mathscr{M}_{f}$ is holonomic if $\mathscr{M}$ is holonomic outside $f^{-1}(0)$. Also, we shall show the existence of $b$-functions, i.e., for a section $u$ of $\mathscr{M}$, there is a nonzero polynomial $b(s)$ and a differential operator $P(s)$ which is a polynomial on $s$ satisfying $P(s) f^{s+1} u=b(s) f^{s} u$.

We use the same technique as in [6].

2.2. Let $s$ be an indeterminate. The sheaf $\mathscr{D}_{X}[s]$ is, by definition, the sheaf of rings $\mathscr{D}_{X} \otimes \mathbb{C}[s]$, where $s$ commutes with the sections of $\mathscr{D}_{X}$. Let $\mathbb{C}[s, t]$ be the ring generated by $s$ and $t$ with the fundamental commutation relation

$$
[t, s]=t \text {. }
$$

We denote by $\mathscr{D}_{X}[s, t]$ the ring $\mathscr{D}_{X} \underset{\mathbb{C}}{\otimes} \mathbb{C}[s, t]$, in which $s$ and $t$ commute with the sections of $\mathscr{D}_{X}$.

Let $\mathscr{M}$ be a coherent $\mathscr{D}_{X}$-Module holonomic outside $f^{-1}(0)$ and $u$ a section of $\mathscr{M}$. Let $\mathscr{J}$ be the Ideal of $\mathscr{D}[s]$ consisting of the $P(s)$ in $\mathscr{D}[s]$ such that

$$
f^{m n-s} P(s) f^{s} u=0
$$

for a sufficiently large $m$.

Note that $f^{m-s} P(s) f^{s}$ belongs to $\mathscr{D}[s]$ for a sufficiently large $m$, and the identity (2.2.1) should be understood to hold in $\mathbb{C}[s] \underset{\mathbb{C}}{\otimes} \mathscr{M}$. We will denote by 
$\mathscr{N}$ the $\mathscr{D}[s]$-Module $\mathscr{D}[s] / \mathscr{J}$ and the modulo class [1] is denoted symbolically by $f^{s} u$. Therefore, $\mathscr{N}$ is generated by $f^{s} u$ as a $\mathscr{D}[s]$-Module.

The following lemma is evident.

Lemma 2.1. The system $\mathscr{N}$ has a structure of a $\mathscr{D}[s, t]$-Module by

$t: P(s) f^{s} u \mapsto P(s+1) f^{s+1} u$.

For any complex number $\lambda, \mathcal{N}(s-\lambda) \mathscr{N}$ is denoted by $\mathcal{N}_{\lambda}$, and $f^{s} u$ modulo $(s-\lambda) \mathscr{N}$ is denoted by $f^{\lambda} u . \mathscr{N}_{\lambda}$ is a $\mathscr{D}_{X^{-}}$-Module generated by $f^{\lambda} u$.

Lemma 2.2. $\mathscr{D} f^{s} u$ and $\mathscr{N}_{\lambda}$ are coherent $\mathscr{D}_{X}$-Modules.

This lemma is an immediate consequence of the following proposition proved in [4]. (See also [8].)

Proposition $2.3([4])$. Let $\mathscr{D}_{m}$ be the sheaf of differential operators of order $\leqq m$. An Ideal $\mathscr{I}$ of $\mathscr{D}_{X}$ is coherent if $\mathscr{I}_{\cap} \mathscr{D}_{m}$ is a coherent $\mathcal{O}_{X}$-Module for any $m$.

2.3. We will take a stratification $\left\{X_{\alpha}\right\}_{\alpha \in A}$ of $X$ such that

$$
S S(\mathscr{M}) \subset \bigsqcup_{\alpha \in A} T_{X_{\alpha}}^{*} X \cup \pi^{-1}\left(f^{-1}(0)\right)
$$

Here, $T_{X_{\alpha}}^{*} X$ signifies the conormal bundle of $X_{\alpha}$.

(2.3.2) Any $X_{\alpha}$ is either disjoint from $f^{-1}(0)$ or contained in $f^{-1}(0)$.

It is clear that there exists such a stratification.

Lemma 2.4. There exists a neighborhood $\Omega$ of $f^{-1}(0)$ such that, for any $X_{\alpha}$ disjoint from $f^{-1}(0), d\left(f \mid X_{\alpha}\right)$ does not vanish at any point in $\Omega \cap X_{\alpha}$.

Proof. If it fails, there exists an analytic path $x(t)$ such that $x(0) \in f^{-1}(0), x(t) \in X_{\alpha}$ for $0<|t| \ll 1$ and that $d\left(f \mid X_{\alpha}\right)$ vanishes at $x(t)$ for $0<|t| \ll 1$. Therefore, $f(x(t))$ is a constant function of $t$, which implies that $f(x(t))=0$. This leads to contradiction. Q.E.D.

Theorem 2.5. On some neighborhood $\Omega$ of $f^{-1}(0), \mathscr{D}\left(f^{s} u\right)$ (resp. $\mathscr{N}_{\lambda}$ ) is a subholonomic (resp. holonomic) $\mathscr{D}_{X}$-Module. (A coherent $\mathscr{D}_{X}$-Module is called holonomic (resp. subholonomic) if the codimension of the characteristic variety is at least $\operatorname{dim} X($ resp. $\operatorname{dim} X-1)$.)

In order to prove this theorem, we note the following proposition.

Proposition 2.6. Let $\mathscr{L}_{1}$ and $\mathscr{L}_{2}$ be two coherent $\mathscr{D}_{X}$-Modules. Suppose that $S S\left(\mathscr{L}_{1}\right) \cap S S\left(\mathscr{L}_{2}\right)$ is contained in the zero section of the cotangent bundle $T^{*} X$. Then $\mathscr{L}_{1} \otimes \mathscr{L}_{2}$ is also a coherent $\mathscr{D}_{X}$-Module and its characteristic variety is contained in

$$
\left\{\left(x, \xi_{1}+\xi_{2}\right) \in T^{*} X ;\left(x, \xi_{1}\right) \in S S\left(\mathscr{L}_{1}\right) \text { and }\left(x, \xi_{2}\right) \in S S\left(\mathscr{L}_{2}\right)\right\} .
$$

Especially, if $\mathscr{L}_{1}$ is holonomic (resp. subholonomic) and $\mathscr{L}_{2}$ is holonomic, then $\mathscr{L}_{1} \otimes \mathscr{L}_{2}$ is holonomic (resp. subholonomic). 
Since $\mathscr{L}_{1} \otimes \mathscr{L}_{2}$ is obtained as the restriction of the system $\mathscr{L}_{1} \hat{\otimes} \mathscr{L}_{2}$ on $X \times X$ onto the diagonal set. (See Proposition 4.7.) This proposition is a consequence of Chapter II, Theorem 3.5.3 and Theorem 3.5.9 of [9].

Now, let us prove Theorem 2.5. We take $\Omega$ as in Lemma 2.4. Since $S S(\mathscr{D} u) \cap S S\left(\mathscr{D} f^{s}\right)$ (resp. $S S(\mathscr{D} u) \cap S S\left(\mathscr{D} f^{\lambda}\right)$ ) is contained in the zero section of $T^{*} X$ on $\Omega-f^{-1}(0), \mathscr{D} f^{s} \otimes \mathscr{D} u$ (resp. $\mathscr{D} f^{\lambda} \otimes \mathscr{D} u$ ) is subholonomic (resp. holonomic) on $\Omega-f^{-1}(0)$. Since there are surjective homomorphisms $\mathscr{D} f^{s} \otimes \mathscr{D} u \supset \mathscr{D}\left(f^{s} \otimes u\right) \rightarrow \mathscr{D}\left(f^{s} u\right)\left(\right.$ resp. $\left.\mathscr{D} f^{\lambda} \otimes \mathscr{D} u \supset \mathscr{D}\left(f^{\lambda} \otimes u\right) \rightarrow \mathscr{N}_{\lambda}\right)$, we can conclude that $\mathscr{D}\left(f^{s} u\right.$ ) (resp. $\mathscr{N}_{\lambda}$ ) is subholonomic (resp. holonomic) on $\Omega-f^{-1}(0)$.

Let $\mathscr{L}$ (resp. $\mathscr{L}^{\prime}$ ) be the sub-Module of $\mathscr{D}\left(f^{s} u\right)$ (resp. $\mathscr{N}_{\lambda}$ ) consisting of all $w$ such that $\mathscr{D} w$ is subholonomic (resp. holonomic). By [4] (cf. [6]), $\mathscr{L}$ (resp. $\mathscr{L}^{\prime}$ ) is subholonomic (resp. holonomic) on $\Omega$. Therefore, $\mathscr{D}\left(f^{s} u\right) / \mathscr{L}$ and $\mathscr{N}_{\lambda} / \mathscr{L}^{\prime}$ are coherent $\mathscr{D}_{x}$-Modules supported in $f^{-1}(0)$. Therefore, by Hilbert's Nullstelensatz, there exists an integer $m$ such that $f^{m} \cdot f^{s} u \in \mathscr{L}$ (resp. $f^{m} \cdot f^{\lambda} u \in \mathscr{L}^{\prime}$ ). Therefore, $\mathscr{D}\left(f^{m} \cdot f^{s} u\right)$ (resp. $\mathscr{D}\left(f^{m} \cdot f^{\lambda} u\right)$ ) is a subholonomic (resp. holonomic) system on $\Omega$. However, $\mathscr{D}\left(f^{m} \cdot f^{s} u\right)$ is isomorphic to $\mathscr{D}\left(f^{s} u\right)$ by the homomorphism $t^{m}$. Hence, it follows that $\mathscr{D}\left(f^{s} u\right)$ is subholonomic.

$\mathscr{D}\left(f^{m} \cdot f^{s} u\right)$ and $\mathscr{D}\left(f^{s} u\right)$ have the same multiplicity at the irreducible components of the characteristic variety of $\mathscr{D}\left(f^{s} u\right)$. Since the multiplicity is an additive quantity, the characteristic variety of $\mathscr{D} f^{s} u / \mathscr{D} f^{m} \cdot f^{s} u$ does not contain any irreducible component of that of $\mathscr{D} f^{s} u$. This implies that $\mathscr{D} f^{s} u / \mathscr{D} f^{m} f^{s} u$ is a holonomic $\mathscr{D}_{X}$-Module.

There exists a surjective homomorphism $\mathscr{D} f^{s} u / \mathscr{D} f^{m} \cdot f^{s} u \rightarrow \mathscr{D}\left(f^{\lambda} u\right) / \mathscr{D}$ $\cdot\left(f^{m} \cdot f^{\lambda} u\right)$, which shows that $\mathscr{D} f^{\lambda} u / \mathscr{D}\left(f^{m} \cdot f^{\lambda} u\right)$ is holonomic. Since $\mathscr{D}\left(f^{m} \cdot f^{\lambda} u\right)$ is holonomic, $\mathscr{D} f^{\lambda} u$ is also holonomic. Thus, Theorem 2.5 is proved.

2.4. Since $\mathscr{N}$ has a structure of a $\mathscr{D}[s, t]$-Module, we can define the $b$-function as in [6]. Recall that the $b$-function is a generator of the ideal of $\mathbb{C}[s]$ consisting of $b(s)$ such that $b(s) \mathscr{N} \subset t \mathscr{N}$. That is equivalent to saying that there exists $P(s) \in \mathscr{D}[s]$ such that $P(s) f^{s+1} u=b(s) f^{s} u$. However, we cannot apply [6] directly in order to prove the existence of nonzero $b$-functions, because $\mathscr{N}$ is not a coherent $\mathscr{D}$-Module in general.

Theorem 2.7. For any point $x_{0} \in f^{-1}(0)$, there exist a nonzero polynomial $b(s)$ of $s$ and $P(s) \in \mathscr{D}[s]_{x_{0}}$ such that

$$
P(s) f^{s+1} u=b(s) f^{s} u \text {. }
$$

Proof. We set $\mathscr{M}^{\prime}=\mathscr{O}_{\mathbb{C}} \hat{\otimes} \cdot \mathscr{H}$. Then $\mathscr{H}^{\prime}$ is a holonomic $\mathscr{D}_{X^{\prime}}$-Module on $X^{\prime}=\mathbb{C} \times X$. We denote by $u^{\prime}$ the section $1 \otimes u$ of $\mathscr{M}^{\prime}$. Set $f^{\prime}(y, x)=y f(x)(y \in \mathbb{C}, x \in X)$. We have

$$
\mathscr{D}_{X^{\prime}}[s] f^{s} u^{\prime}=\mathscr{D}_{X^{\prime}} f^{\prime s} u^{\prime}
$$

In fact, we have

$$
\left(y \frac{\partial}{\partial y}\right)^{m} f^{\prime s} u^{\prime}=s^{m} f^{\prime s} u^{\prime}
$$


Therefore, $\mathscr{N}^{\prime}=\mathscr{D}_{X^{\prime}}[s] f^{\prime s} u^{\prime}$ is subholonomic by Theorem 2.5 and has a structure of $\mathscr{D}_{X^{\prime}}[s, t]$-Module. Therefore, we can apply [6], There exist a polynomial $b(s)$ and a differential operator $P\left(y, x, D_{y}, D_{x}\right)$ defined in a neighborhood of $(y, x)$ $=\left(0, x_{0}\right)$ such that

(2.3) $\quad P\left(y, x, D_{y}, D_{x}\right) f^{\prime s+1} u^{\prime}=b(s) f^{\prime s} u^{\prime}$.

Let $P_{0}$ be the homogeneous component of $P$ of degree -1 with respect to $y$. Then, comparing the degree of homogeneity of (2.3), we have

$$
P_{0} f^{\prime s+1} u^{\prime}=b(s) f^{\prime s} u^{\prime} .
$$

$P_{0}$ has the form

$$
P_{0}=\sum A_{j}\left(x, D_{x}\right)\left(y D_{y}\right)^{j} D_{y} .
$$

Therefore, we have

$$
(s+1) \Sigma s^{j} A_{j}\left(x, D_{x}\right) f^{\prime s} f u^{\prime}=b(s) f^{\prime s} u^{\prime},
$$

which implies

$$
(s+1) \sum s^{j} A_{j}\left(x, D_{x}\right) f^{s+1} u=b(s) f^{s} u \text {. Q.E.D. }
$$

Now, it is easy to see that the canonical homomorphism

$$
\mathscr{N}_{\lambda+1} \rightarrow \mathscr{N}_{\lambda} \quad\left(f^{\lambda+1} u \mapsto f \cdot f^{\lambda} u\right)
$$

is an isomorphism when $b(\lambda) \neq 0$, because we can construct the inverse $f^{\lambda} u \mapsto b(\lambda)^{-1} P(\lambda) f^{\lambda+1} u$.

Therefore, we get the following

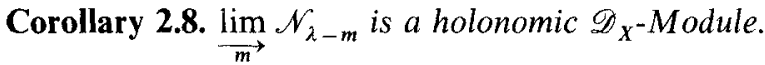

Proposition 2.9. For any coherent $\mathscr{D}_{X}-$ Module $\mathscr{M}, \mathscr{M}_{f}$ is a (coherent) holonomic $\mathscr{D}_{X}$-Module if $\mathscr{M}$ is holonomic outside $f^{-1}(0)$.

Proof. Since $\mathscr{M} \mapsto \mathscr{M}_{f}$ is an exact functor, we may assume without loss of generality that $\mathscr{M}$ is generated by a section $u$.

Since $\mathscr{M}_{f}$ is the quotient of $\underset{m}{\lim } \mathscr{D} f^{-m} u, \mathscr{M}_{f}$ is holonomic. Q.E.D.

\section{§3. Proof of Theorem}

Proposition 2.9 implies Theorem 1.4 almost immediately. First note the following proposition.

Proposition 3.1 ([2], [3]). Let $Y_{1}$ and $Y_{2}$ be two analytic sets. Then there exists a spectral sequence

$$
\mathscr{E}_{2}^{p q}=\mathscr{H}_{\left[Y_{1}\right]}^{p}\left(\mathscr{H}_{\left[Y_{2}\right]}^{q}(\mathscr{M})\right) \Rightarrow \mathscr{H}^{p+q}=\mathscr{H}_{\left[Y_{1} \cap Y_{2}\right]}^{p+q}(\mathscr{M}) .
$$


In particular, if $\mathscr{E}_{2}^{p q}$ are holonomic, then $\mathscr{H}^{p+q}$ are holonomic. Therefore, if Theorem 1.4 is true for $Y_{1}$ and $Y_{2}$, then it is so for $Y_{1} \cap Y_{2}$. Since $Y$ is locally a finite intersection of hypersurfaces, we can reduce the theorem to the case in which $Y$ is a hypersurface by induction. This case is nothing but Proposition 2.9 .

More generally, we have the following theorem. The author is grateful to J.-M. Kantor for kindly pointing out this result.

Theorem 3.1. Let $\mathscr{M}$ be a coherent $\mathscr{D}_{X}$-Module and $Y$ an analytic set of $X$. Suppose that $\mathscr{M}$ is holonomic on $X-Y$. Then $\mathscr{H}_{[X \mid Y]}^{i}(\mathscr{M})$ is coherent and holonomic for any $i$.

Proof. Let $\mathscr{M}^{\prime}$ be the sub-Module of $\mathscr{M}$ consisting of sections $u$ of $\mathscr{M}$ such that $\mathscr{D}_{X} u$ is holonomic. Then, $\mathscr{M}^{\prime}$ is a holonomic system. Since $\mathscr{M}=\mathscr{M}^{\prime}$ outside $X-Y$, the support of $\mathscr{M} / \mathscr{M}^{\prime}$ is contained in $Y$. Therefore, we have $\mathbb{R} \Gamma_{[X \mid Y]}\left(\mathscr{M} / \mathscr{M}^{\prime}\right)$ $=0$, which implies that

$$
\mathbb{R} \Gamma_{[X \mid Y]}(\mathscr{M})=\mathbb{R} \Gamma_{[X \mid Y]}\left(\mathscr{M}^{\prime}\right) .
$$

Thus, replacing $\mathscr{M}$ with $\mathscr{M}^{\prime}$, we may assume that $\mathscr{M}$ is holonomic from the first time. By the exact sequence

$$
0 \rightarrow \mathscr{H}_{[Y]}^{0}(\mathscr{M}) \rightarrow \mathscr{M} \rightarrow \mathscr{H}_{[X \mid Y]}^{0}(\mathscr{M}) \rightarrow \mathscr{H}_{[Y]}^{1}(\mathscr{M}) \rightarrow 0,
$$

and by the isomorphisms

$$
\mathscr{H}_{[X \mid Y]}^{j}(\mathscr{M})=\mathscr{H}_{[Y]}^{j+1}(\mathscr{A}) \quad(j \geqq 1),
$$

this theorem follows from Theorem 1.4. Q.E.D.

\section{$\S 4$. Restriction of $\mathscr{D}_{\mathrm{x}}$-Modules}

4.1. Let $X$ and $Y$ be complex manifolds and $f$ a holomorphic map from $Y$ to $X$. As in [4], we define the sheaf $\mathscr{D}_{Y \rightarrow X}$ (resp. $\mathscr{D}_{X+Y}$ ) by $\mathscr{O}_{Y} \underset{f^{-1} \mathscr{O}_{X}}{\otimes} f^{-1} \mathscr{D}_{X}$ (resp. $\left.f^{-1}\left(\mathscr{D}_{X} \otimes\left(\Omega_{X}^{\operatorname{dim} X}\right)^{\otimes-1}\right) \otimes \Omega_{Y}^{\operatorname{dim} Y}\right)$, where $\Omega_{X}^{j}$ signifies the sheaf of the $j$-forms. The sheaf $\mathscr{D}_{Y \rightarrow X}$ has a structure of right $f^{-1} \mathscr{D}_{X}$-Module by the multiplication from the right. We can endow $\mathscr{D}_{Y \rightarrow X}$ with a structure of left $\mathscr{D}_{X}$-Module as follows. For $v \in \Theta_{Y}, f_{*}(v) \in \mathcal{O}_{Y} \otimes_{f^{-1} \mathbb{C}_{X}} f^{-1} \Theta_{X}$ is given $\Sigma a_{j} \otimes \omega_{j}$ with $a_{j} \in \mathcal{O}_{Y}$ and $\omega_{j} \in \Theta_{X}$. Then $v(b \otimes P)=\Sigma a_{j} b \otimes \omega_{j} P+v(b) \otimes P$.

$\mathscr{D}_{X \leftarrow Y}$ has evidently the structure of left $f^{-1} \mathscr{D}_{X}$-Module. The structure of right $\mathscr{D}_{X}$-Module on $\mathscr{D}_{X}$ induces the structure of left $\mathscr{D}_{X}$-Module on $\mathscr{D}_{X} \otimes_{\mathscr{C}_{X}}\left(\Omega_{X}^{\operatorname{dim} X}\right)^{\otimes-1}$ and hence

$$
\mathscr{O}_{Y} \underset{f^{-1} \mathscr{C}_{X}}{\otimes}\left(\mathscr{D}_{X} \otimes\left(\mathscr{O}_{X}\left(\Omega_{X}^{\operatorname{dim} X}\right)^{\otimes-1}\right)=\mathscr{D}_{Y \rightarrow X} \underset{f^{-1} \mathscr{D}_{X}}{\otimes} f^{-1}\left(\mathscr{D}_{X} \otimes\left(\Omega_{X}^{\operatorname{dim} X}\right)^{\otimes-1}\right)\right.
$$

has a structure of left $\mathscr{D}_{\mathrm{Y}}$-Module. This defines the structure of right $\mathscr{D}_{Y}$-Module on $\mathscr{D}_{X \leftarrow Y}$. Thus, $\mathscr{D}_{Y \rightarrow X}$ is a $\left(\mathscr{D}_{Y}, f^{-1} \mathscr{D}_{X}\right)$-bi-Module and $\mathscr{D}_{X+Y}$ is an $\left(f^{-1} \mathscr{D}_{X}, \mathscr{D}_{Y}\right)$-bi- 
Module. Note that we have

$$
\mathscr{D}_{Y \rightarrow X}=\mathscr{H}_{[Y]}^{\operatorname{dim} X}\left(\mathcal{O}_{Y \times X} \otimes \Omega_{\mathscr{C}_{X}}^{\operatorname{dim} X}\right)
$$

and

$$
\mathscr{D}_{X \leftarrow Y}=\mathscr{H}_{[X]}^{\operatorname{dim} X}\left(\Omega_{Y}^{\operatorname{dim} Y} \otimes_{\mathscr{O}_{Y}}^{\otimes} \mathcal{O}_{Y \times X}\right) .
$$

4.2. Suppose that $Y$ is a submanifold of $X$ of codimension $l$. Then $\mathscr{D}_{Y \rightarrow X}$ and $\mathscr{D}_{X \leftarrow Y}$ are coherent $\mathscr{D}_{X}$-Modules and faithfully flat over $\mathscr{D}_{Y}$. We define for a left $\mathscr{D}_{X}$-Module $\mathscr{M}$

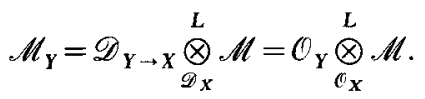

Theorem 4.1. If $\mathscr{M}$ is a holonomic $\mathscr{D}_{X}$-Module, then $\mathscr{T}_{0} r_{k}^{\mathbb{X}_{x}}\left(\mathcal{O}_{Y}, \mathscr{M}\right)=\mathscr{T}_{O} r_{k}^{\mathscr{O} X}$ $\cdot\left(\mathscr{D}_{\mathrm{Y} \rightarrow X}, \mathscr{M}\right)$ is a holonomic $\mathscr{D}_{\mathrm{Y}^{-}}$-Module for any $k$.

This theorem is a consequence of the following propositions.

Proposition 4.2. If $\mathscr{M}$ is a coherent $\mathscr{D}_{X}$-Module whose support is contained in $Y$, then we have

$$
\mathscr{M}=\mathscr{D}_{X+Y} \otimes_{\mathscr{D}_{\mathbf{Y}}} \mathscr{H}_{a m_{\mathscr{D}_{X}}}\left(\mathscr{D}_{X-Y} ; \mathscr{M}\right),
$$

$\mathscr{H}_{\mathrm{O}_{\mathscr{D}_{X}}}\left(\mathscr{D}_{X+Y} ; \mathscr{M}\right)$ is a coherent $\mathscr{D}_{Y}$ Module and $\mathscr{E}_{x} t_{\mathscr{O}_{X}}^{j}\left(\mathscr{D}_{X \leftarrow Y} ; \mathscr{M}\right)=0$ for $j \neq 0$. If moreover, $\mathscr{M}$ is holonomic, so is $\mathscr{H}_{0 n_{\mathscr{D}_{X}}}\left(\mathscr{D}_{X-Y} ; \mathscr{M}\right)$. See [5]. $\begin{array}{lll}\text { Proposition 4.3. } & \mathbb{R} \mathscr{H}_{o m_{\mathscr{O} X}}\left(\mathscr{D}_{X-Y} ; \mathbb{R} \Gamma_{[Y]}(\mathscr{M})\right)[l]=\mathscr{D}_{Y \rightarrow X} \underset{\mathscr{D}_{X}}{\otimes} \mathscr{M} \quad \text { for } \quad \text { any } \\ \mathscr{D}_{X} \text {-Module } \mathscr{M} . & \end{array}$

Proof. Since

$$
\begin{aligned}
& \mathbb{R} \mathscr{H} \operatorname{m}_{\mathscr{D}_{X}}\left(\mathscr{D}_{X-Y} ; \mathbb{R} \Gamma_{[Y]}(\mathscr{M})\right) \\
& =\mathbb{R} \mathscr{H} \operatorname{mon}_{\mathscr{D}_{X}}\left(\mathscr{D}_{X+Y} ; \mathbb{R} \Gamma_{[Y]}\left(\mathscr{D}_{X}\right)\right) \underset{\mathscr{I}_{X}}{\stackrel{L}{\otimes} \mathscr{M},}
\end{aligned}
$$

it is enough to show

$$
\mathbb{R} \mathscr{H}_{\operatorname{am}_{\mathscr{D}_{X}}}\left(\mathscr{D}_{X \leftarrow Y} ; \mathbb{R} \Gamma_{[Y]}\left(\mathscr{D}_{X}\right)\right)[l]=\mathscr{D}_{Y \rightarrow X} .
$$

Set $n=\operatorname{dim} X$. Then, by the definition,

$$
\mathscr{D}_{X+Y}=\mathscr{D}_{X} \stackrel{\mathcal{O}}{X}_{\mathscr{C}_{X}}^{\mathrm{Q}}\left(\Omega_{Y}^{n-l} \otimes\left(\Omega_{X}^{n}\right)^{\otimes-1}\right) .
$$

Hence, we have

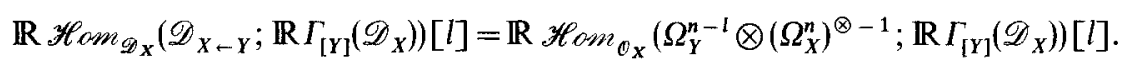

Since $\Omega_{Y}^{n-l} \otimes\left(\Omega_{X}^{n}\right)^{\otimes-1}$ is a coherent $\mathcal{O}_{X}$-Module supported on $Y$, we have

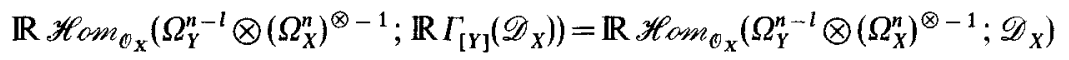


and the last term equals

$$
\mathbb{R} \mathscr{H} \operatorname{com}_{\mathscr{O}_{X}}\left(\Omega_{Y}^{n-l} \otimes\left(\Omega_{X}^{n}\right)^{\otimes-1} ; \mathcal{O}_{X}\right) \underset{\mathscr{C}_{X}}{\stackrel{L}{\otimes}} \mathscr{D}_{X}
$$

Since $\mathscr{E}_{x} t_{\mathcal{O}_{X}}^{j}\left(\mathcal{O}_{Y} ; \mathscr{O}_{X}\right)=\left(\Omega_{Y}^{n-l}\right)^{\otimes-1} \otimes \Omega_{X}^{n}$ for $j=l$ and vanishes for $j \neq l$, we have

$$
\mathbb{R} \mathscr{H}^{0 m_{\mathcal{O}_{X}}}\left(\Omega_{Y}^{n-1} \otimes\left(\Omega_{X}^{n}\right)^{\otimes-1} ; \mathcal{O}_{X}\right)=\mathcal{O}_{Y}[-l] .
$$

Thus we obtain

$$
\left.\mathbb{R} \mathscr{H}^{\cos \mathscr{D}_{X}}{ }_{\left(\mathscr{D}_{X+Y}\right.} ; \mathbb{R} \Gamma_{[Y]}\left(\mathscr{D}_{X}\right)\right)[l]=\mathcal{O}_{Y} \stackrel{\mathscr{O}}{X}_{\mathscr{D}_{X}}^{L}=\mathscr{D}_{Y \rightarrow X} \text {. Q.E.D. }
$$

Now, we can prove Theorem 4.1.

By Theorem $1.4, \mathscr{H}_{[y]}^{k}(\mathscr{M})$ are holonomic when $\mathscr{M}$ is holonomic. Then, by Proposition 4.2, $\mathbb{R} \mathscr{H}_{\mathrm{om}_{\mathscr{O}_{X}}}\left(\mathscr{D}_{X-Y} ; \mathbb{R} \Gamma_{[Y\}}(\mathscr{M})\right)$ has holonomic $\mathscr{D}_{Y}$-Modules as cohomologies, Hence, Theorem 4.1 follows immediately from Proposition 4.3.

4.3. Suppose that $f: Y \rightarrow X$ is a holomorphic map.

Theorem 4.4. If $\mathscr{M}$ is a holonomic $\mathscr{D}_{X}$-Module, then

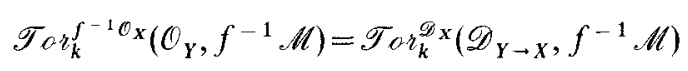

is a holonomic $\mathscr{D}_{\mathrm{Y}}$-Module.

Proof. Let $\mathscr{N}$ be the holonomic system $\mathcal{O}_{Y} \hat{\otimes} \mathscr{M}$. Then $\mathscr{N}$ is a holonomic $\mathscr{D}_{Y \times X^{-}}$

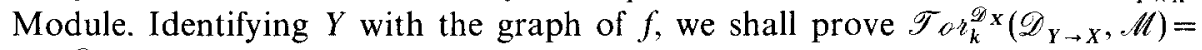
$\mathscr{T}_{0} \mathscr{T}_{k}^{\mathscr{D} \times X}\left(\mathscr{D}_{Y \rightarrow Y \times X}, \mathcal{N}\right)$. This implies immediately the desired result.

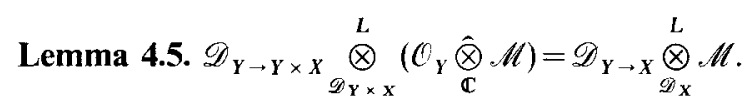

Proof. Let $p_{1}$ and $p_{2}$ be the projection from $Y \times X$ onto $Y$ and $X$, respectively.

$$
\mathscr{O}_{Y} \underset{\mathbb{C}}{\otimes} \mathscr{M}=\mathscr{D}_{Y \times X} \underset{p_{1}^{-1} \mathscr{Q}_{Y} \otimes p_{2}^{-1} \mathscr{D}_{X}}{\otimes}\left(p_{1}^{-1} \mathscr{O}_{Y} \otimes p_{2}^{-1} \mathscr{M}\right) .
$$

Thus, we have

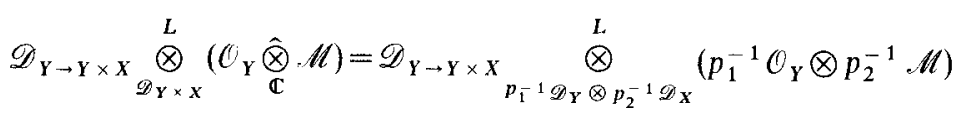

$$
\begin{aligned}
& =\left(\mathscr{D}_{Y \rightarrow Y \times X} \stackrel{L}{\otimes} p_{p_{1}^{-1} \mathscr{D}_{Y}}^{\otimes} p_{1}^{-1} \mathscr{O}_{Y}\right) \underset{p_{2}^{-1} \mathscr{P}_{X}}{\stackrel{L}{\otimes}} p_{2}^{-1} \mathscr{M} .
\end{aligned}
$$

Thus, it is enough to show

$$
\mathscr{D}_{Y \rightarrow Y \times X} \stackrel{L}{\underset{p_{1}^{-1} \mathscr{D}_{Y}}{\otimes}} p_{1}^{-1} \mathscr{O}_{\mathbf{Y}}=\mathscr{D}_{Y \rightarrow X} .
$$

It is easy to see

$$
\mathscr{D}_{Y \times X} \underset{p_{1}^{-1} \mathscr{D}_{Y}}{\stackrel{L}{\otimes}} p_{1}^{-1} \mathcal{O}_{Y}=\mathcal{O}_{Y \times X} \underset{p_{2}^{-1} \mathscr{O}_{X}}{\otimes} p_{2}^{-1} \mathscr{D}_{X}
$$


We have

$$
\begin{aligned}
& \mathscr{D}_{Y \rightarrow Y \times X} \underset{p_{1}^{-1} \mathscr{D}_{Y}}{\otimes} p_{1}^{-1} \mathcal{O}_{Y}=\left(\mathcal{C}_{Y_{C_{Y}}} \underset{C_{X}}{\otimes} \mathscr{D}_{Y \times X}\right) \underset{p_{1}^{-1} \mathscr{D}_{Y}}{\otimes} p_{1}^{-1} \mathcal{O}_{Y} \\
& =\mathcal{O}_{Y} \underset{\mathscr{O}_{Y \times X}}{\otimes}\left(\mathscr{D}_{Y \times X} \underset{p_{1}^{-1} \mathscr{P}_{Y}}{\otimes} p_{1}^{-1} \mathcal{O}_{Y}\right) \\
& =\mathscr{O}_{Y} \underset{\mathcal{O}_{Y \times X}}{\otimes}\left(\mathcal{O}_{Y \times X} \underset{p_{2}^{-1} \mathcal{O}_{X}}{\otimes} p_{2}^{-1} \mathscr{D}_{X}\right)=\mathcal{O}_{Y} \underset{f^{-1} \mathscr{O}_{X}}{\otimes} f^{-1} \mathscr{D}_{X}=\mathscr{D}_{Y \rightarrow X} \text {. Q.E.D. }
\end{aligned}
$$

4.4. We shall prove here the tensor products of two holonomic systems are holonomic.

Theorem 4.6. Let $\mathscr{M}$ and $\mathscr{N}$ be two holonomic $\mathscr{D}_{X}$-Modules; then $\mathscr{T}_{0} r_{k}^{\mathbb{O}}(\mathscr{M}, \mathscr{N})$ is a holonomic $\mathscr{D}_{X}$-Module for any $k$.

Proof. First we shall prove

Proposition 4.7. For two $\mathscr{D}_{X}$-Modules $\mathscr{M}$ and $\mathcal{N}$, we have

$$
\mathscr{M} \stackrel{L}{\otimes} \mathscr{O} \mathcal{N}=\mathscr{D}_{X \rightarrow X \times X} \underset{\mathscr{O}_{X \times X}}{\otimes}(\mathscr{M} \hat{\otimes} \mathscr{N}),
$$

where

$$
\mathscr{M} \hat{\otimes} \mathscr{N}=\mathscr{D}_{X \times X} \underset{p_{1}^{-1} \mathscr{D}_{X} \otimes p_{2}^{-1} \mathscr{D}_{X}}{\otimes}\left(p_{1}^{-1} \mathscr{M} \otimes p_{2}^{-1} \mathscr{N}\right)
$$

with the first and the second projections $p_{1}$ and $p_{2}$ from $X \times X$ onto $X$.

Proof. Since

$$
\mathscr{D}_{X \times X}=\mathcal{O}_{X \times X}{ }_{p_{1}^{-1} \mathscr{O}_{X} \otimes p_{2}^{-1} \mathscr{O}_{X}}\left(p_{1}^{-1} \mathscr{D}_{X} \otimes p_{2}^{-1} \mathscr{D}_{X}\right),
$$

we have

$$
\mathscr{M} \hat{\otimes} \mathscr{N}=\mathcal{O}_{X \times X} \underset{p_{1}^{-1} \mathscr{C}_{X} \otimes p_{2}^{-1} \mathscr{O}_{X}}{\otimes}\left(p_{1}^{-1} \mathscr{M} \otimes p_{2}^{-1} \mathcal{N}\right) .
$$

Therefore,

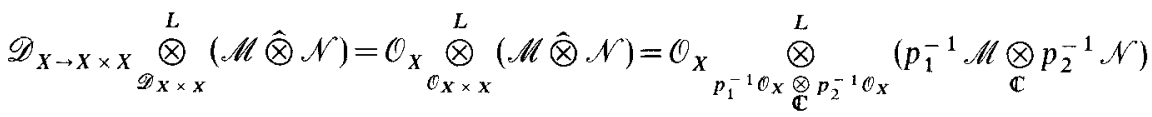

$$
\begin{aligned}
& =\stackrel{L}{\mathscr{M}} \underset{\mathcal{O}_{x}}{\otimes} \mathscr{N} . \quad \text { Q.E.D. }
\end{aligned}
$$

Theorem 4.6 is a consequence of this proposition and Theorem 4.1.

4.5. We know that $\mathscr{E} x t_{\mathscr{Q}_{X}}^{J}\left(\mathscr{M} ; \mathscr{D}_{X}^{\infty} \otimes_{\mathscr{D}_{X}} \mathscr{N}\right)$ is a constructible sheaf for any holonomic $\mathscr{D}_{X}$-Modules $\mathscr{M}$ and $\mathscr{N}$ [5]. Here a sheaf $\mathscr{F}$ is called constructible if there is a stratification of $X$ on each of whose strata $\mathscr{F}$ is locally constant of finite rank. $\mathscr{D}_{X}^{\infty}$ is the sheaf of the differential operators of infinite order. Therefore, in particular, $\mathscr{H}_{\mathfrak{s}_{\mathscr{O}_{X}}}(\mathscr{M} ; \mathscr{N})$ has a finite-dimensional stalk at each point. Furthermore, by using the previous results, we can prove the following results. 
Theorem 4.8. Let $\mathscr{M}$ and $\mathscr{N}$ be two holonomic $\mathscr{D}_{X}$-Modules. Then $\mathscr{E}_{x} t_{\mathscr{D}_{X}}^{j}(\mathscr{M} ; \mathscr{N})$ is a constructible sheaf for any $j$.

Proof. This is a consequence of Lemma 1.8 because and

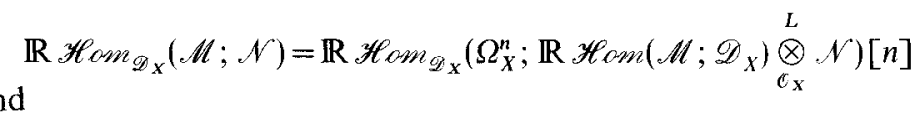

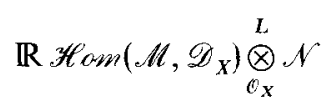

has holonomic $\mathscr{D}_{X}$-Modules as cohomologies. Therefore the theorem follows from the result in [5]. Q.E.D.

\section{References}

1. Bernstein, I.N.: The analytic continuation of generalized functions with respect to a parameter. Functional Anal. Appl. 6, 26-40 (1972)

2. Grothendieck, A.: Cohomologie Locale des Faisceaux Cohérents et Théorèmes de Lefschetz Locaux et Globeaux (SGA2). Amsterdam: North-Holland Publ. Co. 1968

3. Hartshorne, R.: Local Cohomology, Lecture Notes in Math., 41. Berlin-Heidelberg-New York: Springer 1967

4. Kashiwara, M.: An algebraic study of systems of partial differential equations, local theory of differential operators (Master's thesis). Sugakushinkokai (in Japanese), 1970

5. Kashiwara, M.: On the maximally overdetermined system of linear differential equations, I. Publ. R.I.M.S., Kyoto Univ. 10, 563-579 (1975)

6. Kashiwara, M.: $B$-functions and holonomic systems, rationality of roots of $b$-functions. Inventiones Math. 38, 33-53 (1976)

7. Kashiwara, M., Kawai, T.: On the holonomic systems of micro-differential equations, III. in press (1978)

8. Le Jeune-Jalabert, M., Malgrange, B., Boutet de Monvel: Séminaire "Opérateurs différentiels et pseudo-différentiels", I. II, III, IV, Université Scientifique et Médical de Grenoble, Laboratoire de Math. Pures Associé au C.N.R.S., 1975-1976

9. Sato, M., Kawai, T., Kashiwara, M.: Microfunctions and pseudodifferential equations, Lecture Notes in Math. Berlin-Heidelberg-New York: Springer 287. 265-529 (1973)

Received October 24, 1977/Revised June 19, 1978 